\title{
Automatic Segmentation of Prostate Magnetic Resonance Imaging Using Generative Adversarial Networks
}

\section{CURRENT STATUS: POSTED}

Research Square

Wei Wang

Tongji Hospital of Tongji University School of Medicine

Mingang Wang

Huashan Hospital of Fudan University

Xiaofen Wu

Tongji Hospital of Tongji University School of Medicine

Xie Ding

School of Wonders information company

Xuexiang Cao

School of Wonders information company

Lei Wang

Tongji Hospital of Tongji University School of Medicine

Jingyi Zhang

School of Wonders information company

Peijun Wang

Tongji Hospital of Tongji University School of Medicine

$\checkmark$ pjwangtongji@sina.comCorresponding Author

ORCiD: https://orcid.org/0000-0002-4012-9234

DOI:

10.21203/rs.2.12243/v1

\section{SUBJECT AREAS}

Nuclear Medicine \& Medical Imaging

\section{KEYWORDS}

Automatic segmentation, Generative adversarial networks, Magnetic resonance imaging, Prostate 
Abstract

Background: Automatic and detailed segmentation of the prostate using magnetic resonance imaging (MRI) plays an essential role in prostate imaging diagnosis. However, the complexity of the prostate gland hampers accurate segmentation from other tissues. Thus, we propose the automatic prostate segmentation method SegDGAN, which is based on a classic generative adversarial network (GAN) model.

Methods: The proposed method comprises a fully convolutional generation network of densely connected blocks and a critic network with multi-scale feature extraction. In these computations, the objective function is optimized using mean absolute error and the Dice coefficient, leading to improved accuracy of segmentation results and correspondence with the ground truth. The common and similar medical image segmentation networks U-Net, fully convolution network, and SegAN were selected for qualitative and quantitative comparisons with SegDGAN using a 220-patient dataset and the publicly available dataset PROMISE12. The commonly used segmentation evaluation metrics Dice similarity coefficient (DSC), volumetric overlap error (VOE), average surface distance (ASD), and Hausdorff distance (HD) were also used to compare the accuracy of segmentation between these methods.

Results: SegDGAN achieved the highest DSC value of $91.66 \%$, the lowest VOE value of $23.47 \%$ and the lowest ASD values of $0.46 \mathrm{~mm}$ with the clinical dataset. In addition, the highest DCS value of $88.69 \%$, the lowest VOE value of $23.47 \%$, the lowest ASD value of $0.83 \mathrm{~mm}$, and the lowest HD value of $11.40 \mathrm{~mm}$ was achieved with the PROMISE12 dataset.

Conclusions: Our experimental results show that the SegDGAN model outperforms other segmentation methods

Keywords: Automatic segmentation, Generative adversarial networks, Magnetic resonance imaging, Prostate

\section{Full-text}

Due to technical limitations, full-text HTML conversion of this manuscript could not be completed. However, the manuscript can be downloaded and accessed as a PDF from the Manuscript Files section 
below.

Figures

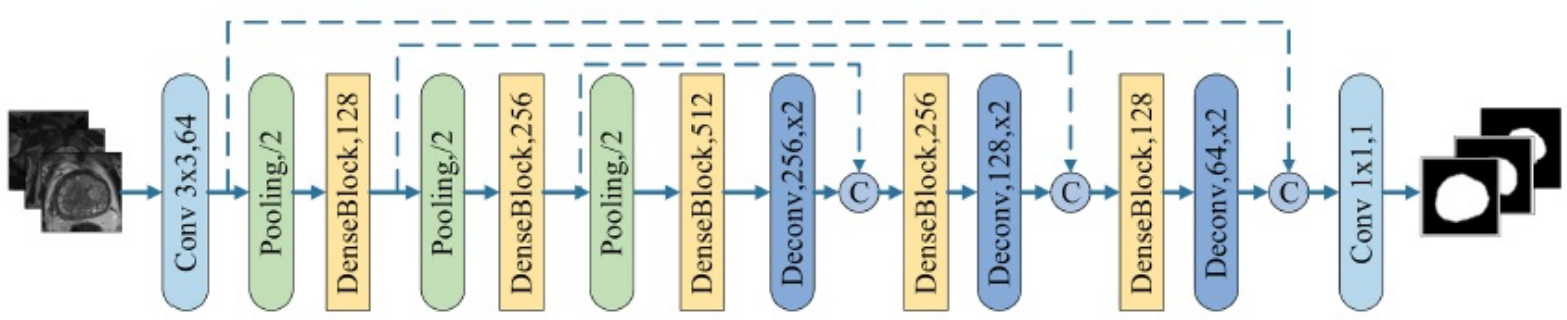

Figure 1

The architecture of generator $\mathrm{G}$. it comprises a down-sampling path with three dense blocks and an up-sampling path with two dense blocks; blue circles represent concatenation in the network. Blue dotted arrows indicate bypass connections of the feature maps between coupled encoders and decoders.

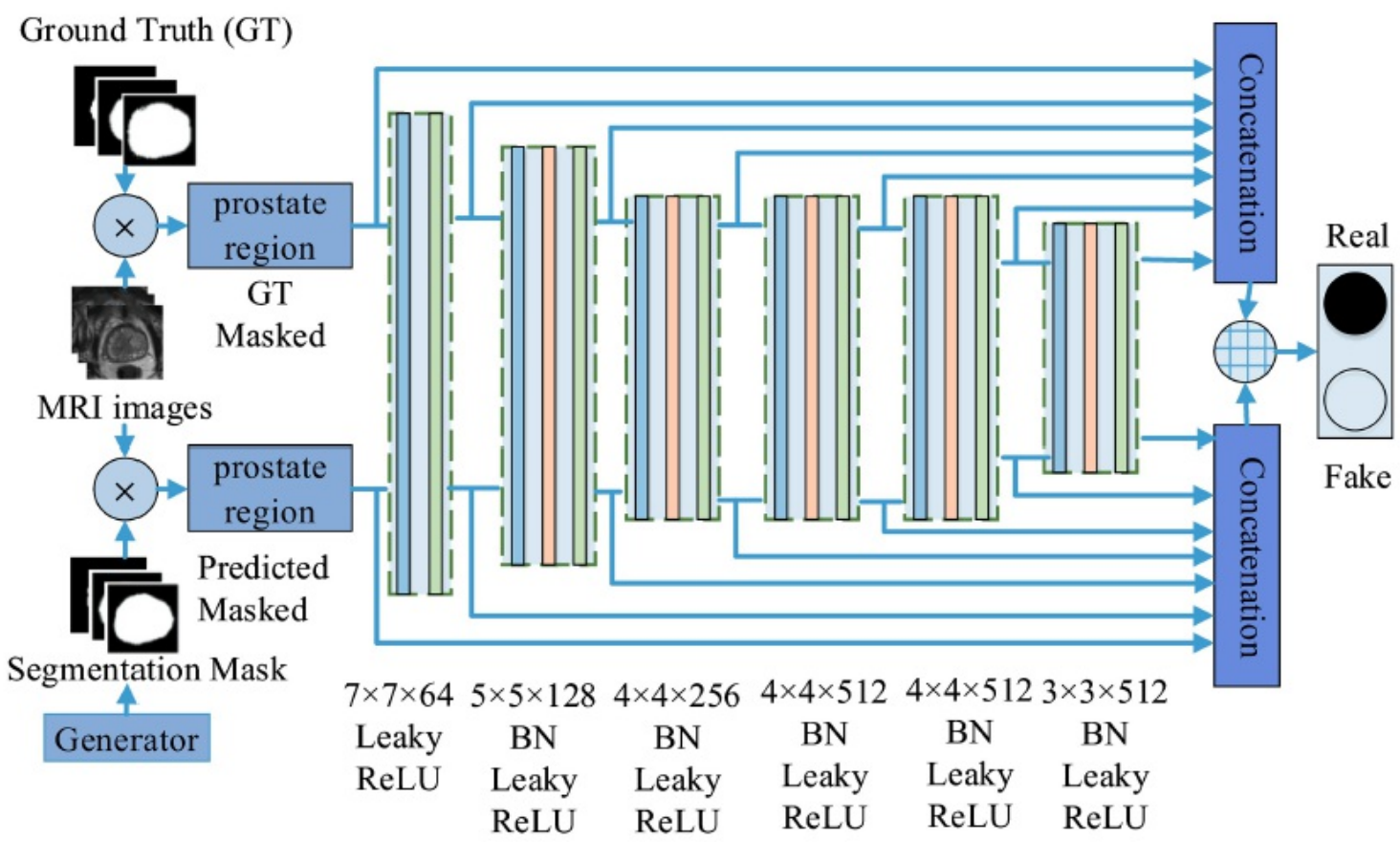

Figure 2

The architecture of discriminator D. All layers comprise convolutional, batch normalization (BN), and leaky ReLU layers. BN is not included in the rst layer. Masked images are generated by multiplying label images with input images in a pixel-wise order. 


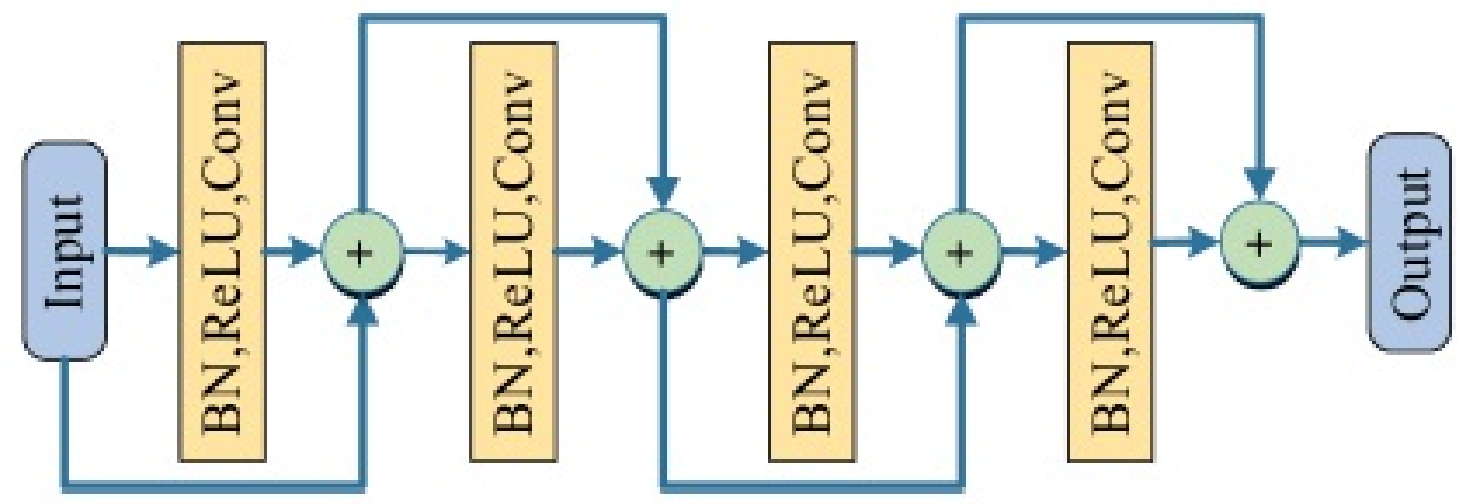

Figure 3

The dense block structure. it is adopted in Generator G. Green circles represent concatenation.

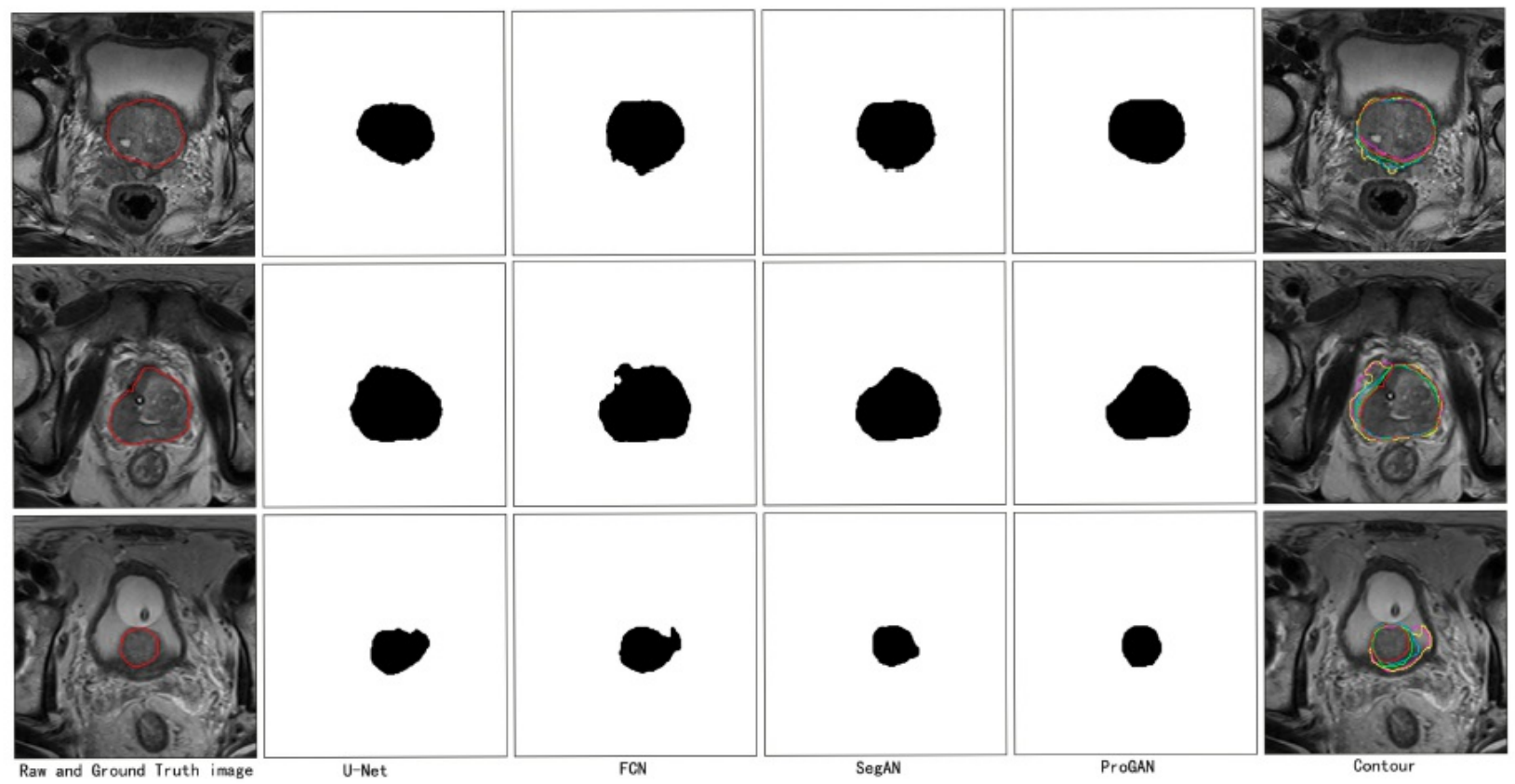

Figure 4

Qualitative segmentation results of a prostate gland. these results from our clinical dataset at the center (upper), apex (middle), and base (lower); the rst column shows raw MRI images with ground truth of the prostate gland. Columns 2-5 correspond with segmentation analyses using U-Net, FCN, SegAN, and SegDGAN models, respectively. In the nal column, ve contours superimposed on the raw MR images; red contour denotes the ground truth, and pink, yellow, blue, and green contours denote the segmentation results from U-Net, FCN, SegAN, and SegDGAN, respectively. 


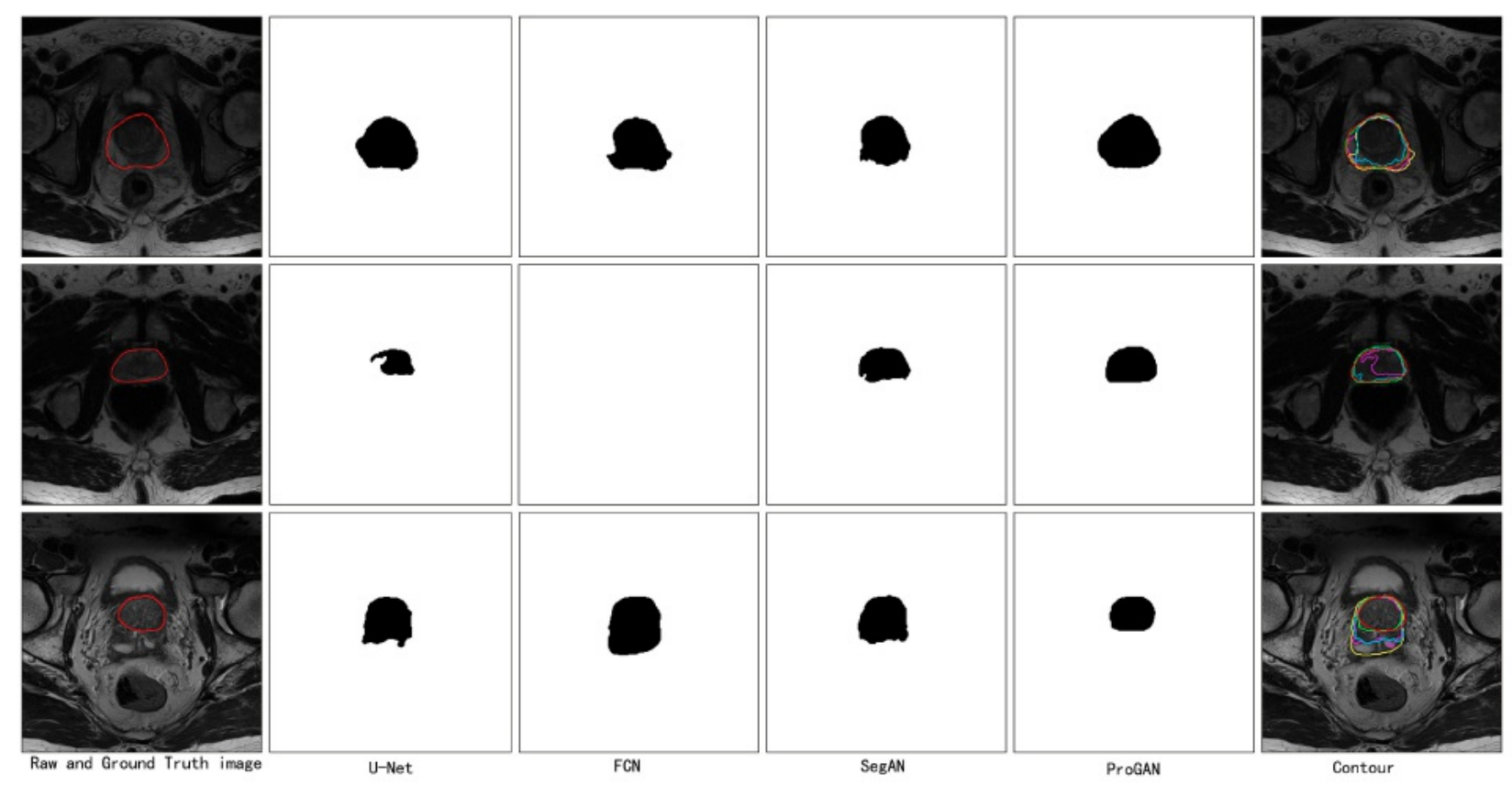

Figure 5

Qualitative segmentation results. the comparable qualitative results of U-Net, FCN, SegAN, and SegDGAN model analyzes using the public dataset PROMISE12.
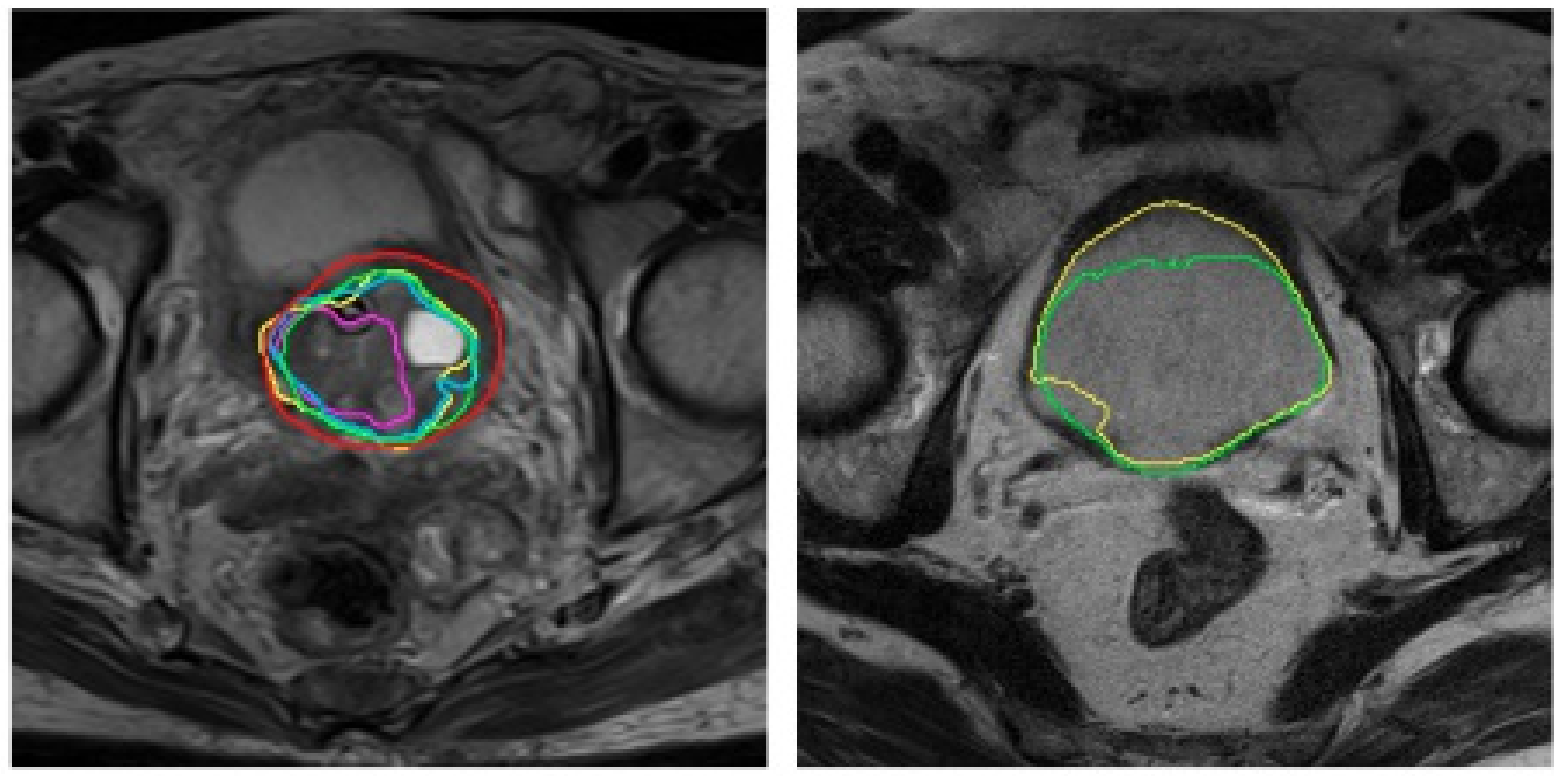

Figure 6

Segmentation results of the worst case. 